\title{
Function of Adipose-Derived Mesenchymal Stem Cells in Monocrotaline-Induced Pulmonary Arterial Hypertension through miR-191 via Regulation of BMPR2
}

\author{
Caixin Zhang $\mathbb{D}^{1}{ }^{1}$ Pengbo Wang, ${ }^{1}$ Anaz Mohammed, ${ }^{1}$ Zhewen Zhou $\mathbb{D},{ }^{2}$ \\ Shuwen Zhang, ${ }^{1}$ Songshi Ni $\mathbb{D},{ }^{1}$ and Zhiyuan Tang $\mathbb{D}^{1}$ \\ ${ }^{1}$ Department of Respiratory and Critical Care Medicine, Affiliated Hospital of Nantong University, Nantong, 226001, Jiangsu, China \\ ${ }^{2}$ Department of Dermatology, Affiliated Hospital of Nantong University, Nantong, 226001, Jiangsu, China
}

Correspondence should be addressed to Songshi Ni; jsntnss@163.com and Zhiyuan Tang; tina2951@sina.com

Received 27 November 2018; Revised 25 February 2019; Accepted 12 March 2019; Published 16 April 2019

Academic Editor: Christina Pabelick

Copyright (c) 2019 Caixin Zhang et al. This is an open access article distributed under the Creative Commons Attribution License, which permits unrestricted use, distribution, and reproduction in any medium, provided the original work is properly cited.

Pulmonary arterial hypertension (PAH) is a serious condition. However, prevailing therapeutic strategies are not effective enough to treat PAH. Therefore, finding an effective therapy is clearly warranted. Adipose-derived mesenchymal stem cells (ASCs) and ASCsderived exosomes (ASCs-Exos) exert protective effects in $\mathrm{PAH}$, but the underlying mechanism remains unclear. Using a coculture of ASCs and monocrotaline pyrrole (MCTP)-treated human pulmonary artery endothelial cells (HPAECs), we demonstrated that ASCs increased cell proliferation in MCTP-treated HPAECs. Results showed that ASCs-Exos improved proliferation of both control HPAECs and MCTP-treated HPAECs. In addition, by transfecting ASCs with antagomir we observed that low exosomal miR-191 expression inhibited HPAECs proliferation whereas the agomir improved. Similar results were observed in vivo using a monocrotaline (MCT)-induced PAH rat model following ASCs transplantation. And ASCs transplantation attenuated MCTinduced PAH albeit less than the antagomir treated group. Finally, we found that miR-191 repressed the expression of bone morphogenetic protein receptor 2 (BMPR2) in HPAECs and PAH rats. Thus, we conjectured that miR-191, in ASCs and ASCsExos, plays an important role in PAH via regulation of BMPR2. These findings are expected to contribute to promising therapeutic strategies for treating PAH in the future.

\section{Introduction}

It is considered to be pulmonary arterial hypertension (PAH) when the mean pulmonary artery pressure (mPAP) is above $25 \mathrm{mmHg}$ at rest [1]. As the disease progresses, pulmonary vascular resistance increases, leading to right heart failure and mortality [2]. Vascular remodeling is the key pathological feature of $\mathrm{PAH}$, characterized by endothelial dysfunction, activation of fibroblasts, and smooth muscle cells. Endothelial dysfunction included suppression of normal apoptosis and excessive proliferation of endothelial cells [3]. So far, the modern therapy has improved clinical state and extended life by a few years, but the vascular changes remain progressive [4]. And no effective therapy that can either terminate or reverse the hyperplasia of human pulmonary artery endothelial cells (HPAECs) is available.
Mesenchymal stem cells (MSCs) are major members of stem cells that were first discovered in bone marrow. Human adipose is an abundant and accessible source of adiposederived mesenchymal stem cells (ASCs) [5]. ASCs are capable of reducing inflammation in damaged tissues, improving angiogenesis, and reducing apoptosis due to their paracrine secretory potential, mitochondrial transfer, and secretion of exosomes [6-9]. Previous studies have shown that MSCs can relieve the development of PAH in animal models [10, 11]. However, no relevant study has been carried out to explore the contribution of ASCs in monocrotaline pyrrole (MCTP)treated HPAECs and monocrotaline (MCT)-induced PAH rat model.

Exosomes are a type of extracellular vesicles between 50 and $150 \mathrm{~nm}$ in size, secreted by almost all types of cells, which are widely present in various body fluids [12]. Exosomes are 
vesicles encapsulated by a lipid bilayer containing biologically active substances such as proteins, microRNAs (miRNAs), long noncoding RNAs (lncRNAs), and rRNAs [13-15]. Recent studies have found that ASCs-derived exosomes (ASCs-Exos) can mimic biological functions of mother cells. ASCs-Exos are being used to alleviate tissue damage and treat incurable diseases, which has broad prospects for development [1618]. However, the effects of ASCs-Exos on HPAECs and the underlying mechanisms are rarely investigated.

MicroRNAs (miRNAs) belong to a class of endogenous single-stranded, noncoding RNAs of approximately 22 nucleotides that repress mRNA translation or stability by binding to the $3^{\prime}$ UTR of the target gene. In mammals, miRNAs are involved in more than $60 \%$ of all protein-coding processes $[19,20]$. Among the various RNAs present in exosomes, miRNAs constitute $76.2 \%$ of the total RNAs, and miR191 is one of the most abundant miRNAs in ASCs-Exos [21]. A recent study has shown elevated miR-191 in the circulation of $\mathrm{PAH}$ patients [22]. However, its function in $\mathrm{PAH}$ remains obscure. In this study, we aimed to investigate the effects of exosomal miR-191 on the growth of MCTP-treated HPAECs as well as the underlying mechanism. Furthermore, we also explored the function of ASCs transfected with miR-191 in the development of PAH in MCT-induced PAH rats.

\section{Materials and Methods}

2.1. Reagents and Antibodies. The antibodies of CD9, CD63, $\mathrm{CD} 81$, and bone morphogenetic protein receptor 2 (BMPR2) were obtained from Abcam (Cambridge, MA, USA). Monocrotaline was purchased from the Fluorochem (Hadfield, Derbyshire, UK). The agomir and antagomir were obtained from Biomics Biotech (Nantong, China).

2.2. Animals. Thirty-five male Sprague-Dawley rats $(240 \pm$ $30 \mathrm{~g}$ ) were provided by the Laboratory Animal Center of Nantong University. All rats were fed in a well-ventilated room with 12-hour light and dark cycle, with standard water and food ad libitum. All courses followed the Guidelines of Chinese Council on Animal Research and the Guidelines of Animal Care to minimize the suffering of animals. This study has been approved by the Administration Committee of Experimental Animals, Jiangsu Province, China (Approval ID: SYXK (SU) 2012-0031).

2.3. Isolation and Culture of Cells. Human ASCs (hASCs) were isolated and cultured as described by Bradley et al. [23]. Briefly, the adipose tissue was collected from a 25 -year-old male's abdomen, washed 3 times with phosphate buffered saline (PBS) (Hyclone, Utah, USA). This work was approved by Ethics Committee of Affiliated Hospital of Nantong University (Examination number: 2018-K020). The fascia and small blood vessels were removed and moistened with $1 \%$ Collagenase I (Biosharp, Hefei, China) for 2 hours; the stem cells were then placed in culture flasks with DMEM/High Glucose medium (Hyclone) supplemented with 10\% fetal bovine serum (Gibco, Carlabad, USA) and incubated at $37^{\circ} \mathrm{C}$. Initially the medium was changed after 72 hours, and thereafter the medium was changed every 2 days. FACS was used to characterize the ASCs. Osteogenic differentiation and adipogenic differentiation assays were performed by the cells of passage 4 . ASCs from passages 3-5 were used for the subsequent experiments.

HPAECs were purchased from BeNa Culture Collection (BeNa, Beijing, China). The cells were maintained in DMEM/ High Glucose medium supplemented with $10 \%$ fetal bovine serum at $37^{\circ} \mathrm{C}$ in a humidified chamber, 5\% $\mathrm{CO}_{2}, 95 \%$ air atmosphere.

2.4. Isolation and Characterization of ASCs-Exos. First, ASCs were cultured in medium supplemented with $10 \%$ serum for 24 hours. Then serum-free medium was used instead, and the cells were incubated for another 24 hours. Exosomes were collected from each conditioned medium by ultracentrifugation at $150,000 \mathrm{~g}$ for 2 hours at $4^{\circ} \mathrm{C}$ (CS150GXL, Hitachi, Japan). Then exosomes were washed once by PBS and they were ultracentrifuged again. Exosomes were suspended in $100 \mu \mathrm{l}$ PBS. Twenty microliters exosomes were loaded onto a formvar/carbon-coated grid, negatively stained with 3\% aqueous phosphotungstic acid for 1 minute and observed by transmission electron microscope (TEM, JEM-1230, JEOL, Tokyo, Japan). BCA Protein Assay Kit (Biosharp) was used to assess the exosomes protein concentration.

2.5. Exosomes Labeling. For the exosome-labeling experiments, purified exosomes were fluorescently labeled using PKH67 Green Fluorescent Cell Linker Mini Kit (SigmaAldrich, USA). Simply, labeled exosomes were collected by ultracentrifugation as described above and resuspended in PBS. HPAECs were seeded and incubated for 1 hour with PKH67-labeled exosomes. Then, the cells were fixed with $4 \%$ paraformaldehyde and washed 3 times with PBS. Nuclei were incubated with DAPI (Beyotime, Shanghai, China) for 10 minutes and washed 3 times with PBS. Images were captured by the Olympus Microscope (Olympus, Japan).

2.6. Preparation of MCTP. MCT was converted to MCTP using tetrabromo-1,2-benzoquinone (Sigma-Aldrich) [24]. The product was stored in N, N-dimethylformamide (DMF) at $-20^{\circ} \mathrm{C}$ prior to use.

2.7. Cell Viability Assay. Cell viability was measured by CCK-8 assay (Beyotime). HPAECs were prepared in 96well cell plates. After 24-hour of incubation, the culture medium was removed and HPAECs were incubated with various concentrations of MCTP $(0-120 \mu \mathrm{g} / \mathrm{ml})$ or vehicle (DMF with the identical dilution rate as MCTP) for different time (0-72h). $10 \mu \mathrm{l}$ CCK-8 solution was added to each well, and the viability was measured at $450 \mathrm{~nm}$ by an enzyme linked immunosorbent assay reader (Bio Tek, Winooski, USA).

2.8. Overexpression and Knockdown of miR-191 in ASCs and ASCs-Exos. ASCs were starved in serum-free medium for 24 hours and then transfected with miR-191 agomir (50 nM), miR-191 antagomir (100 nM), agomir negative control (agomir NC) (50 nM), and antagomir negative control (antagomir NC) (50 nM), respectively, using Lipofectamine 
TABLE 1: Sequences of synthesized miR-191 oligonucleotides.

\begin{tabular}{lc}
\hline Oligonucleotides & \multicolumn{1}{c}{ Sequences $\left(5^{\prime}\right.$ to $\left.3^{\prime}\right)$} \\
\hline miR-191 agomir & CAACGGAAUCCCAAAAGCAGCUG \\
& CAGCUGCUUUUGGGAUUCCGUUG \\
miR-191 antagomir & CAGCUGCUUUUGGGAUUCCGUUG \\
agomir NC & UCACAACCUCCUAGAAAGAGUAGA \\
& UCUACUCUUUCUAGGAGGUUGUGA \\
antagomir NC & UCUACUCUUUCUAGGAGGUUGUGA \\
\hline
\end{tabular}

2000 (Thermo Fisher, Carlsbad, USA) transfection reagent according to the manufacturer's instructions. Twenty-four hours later, serum-free medium was replaced by completed medium. Then exosomes were collected by the method mentioned above, and RT-PCR was used to verify the expression of miR-191. The agomir and antagomir sequences used are shown in Table 1.

2.9. RT-PCR Assay. Total RNA was extracted from cells by TRIzol Reagent (Ambion, Carlsbad, USA) and reversetranscripted by a Reverse Transcriptase kit (Thermo Fisher) according to the manufacturer's protocol. The transcripts were quantified with SYBR Green (Vazyme, Nanjing, China) on a qTOWER 2.2 RT-PCR apparatus (Analytik Jena, Jena, Germany). The relative levels of cellular miRNA were quantified with the $2^{-\Delta \Delta C t}$ after normalizing to U6. The primers used for RT-PCR were designed by RiboBio (Guangzhou, China): Bulge-Loop ${ }^{\mathrm{TM}}$ miRNA qRT-PCR Primer Sets.

2.10. Coculture of ASCs and HPAECs. Coculture was performed using transwell chambers with $3.0-\mu \mathrm{m}$ pore size. The upper chamber was seeded with $1 \times 10^{5}$ ASCs, while $1 \times$ $10^{4}$ HPAECs were seeded in the lower chamber. Twenty-four hours later, HPAECs were fixed in methanol for 10 minutes, stained with crystal violet for another 15 minutes, then washed 3 times with PBS. Digital images were photographed under a microscope.

2.11. Western Blot. The protein extracts were obtained by using RIPA buffer (Beyotime), and protein concentration was determined by the BCA kit. The membrane was blocked with 5\% fat-free milk (Yili, Hohhot, China) for 2 hours at room temperature (RT) and then incubated with the following primary antibodies at $4^{\circ} \mathrm{C}$ overnight. The dilutions of antibodies were anti-CD9 (1:1000), anti-CD63 (1:500), anti-CD81 (1:1000), and anti-BMPR2 (1:500). Samples were normalized to GAPDH (1:3000; Abways, Shanghai, China). The secondary antibodies were incubated for 2 hours at RT. Then protein bands were detected by ChemiDoc XRS + system (Bio-Rad, USA).

2.12. Rat PAH Model and ASCs Injection. Rats were given a single intraperitoneal injection of MCT $(60 \mathrm{mg} / \mathrm{kg})$ to induce $\mathrm{PAH}$, and the control group was treated with saline. After two weeks, the seven groups of rats were treated as follows: none (control group), saline (MCT group), ASCs (MCT + ASC group), miR-191 agomir transfected stem cells (MCT
+ agomir group), miR-191 antagomir transfected stem cells (MCT + antagomir group), agomir NC cells (MCT + agomir NC group), and antagomir NC cells (MCT + antagomir NC group). The number of cells injected was $3 \times 10^{6}$. At the end of the fourth week, all rats followed right ventricle intubation and the right ventricular systolic pressure (RVSP) was measured.

2.13. Measurement of RVSP, Right Ventricle Hypertrophy. All rats were anesthetized with $10 \%$ chloral hydrate $(30 \mathrm{ml} / \mathrm{kg}$, i.p.). Rats were kept in a supine position, the right jugular vein was isolated, and a PE-50 polyethylene tube filled with heparin saline was inserted into the jugular vein. The catheter was then further inserted into the right ventricle, and systolic pressure was recorded by the BIOPAC multilead physiography (BIOPAC Systems, Santa Barbara, USA). Then the hearts were collected, and the right ventricle and left ventricle + septum were carefully separated and weighted. The weight ratio of $\mathrm{RV} / \mathrm{LV}+\mathrm{S}$ was calculated.

2.14. Vascular Morphology of Distal Pulmonary Arteries. The left upper lobe was obtained from each group and fixed with $10 \%$ formalin. H\&E staining was used for histological observations. Six images of pulmonary artery (external diameter 50 200 $\mu \mathrm{m}$ ) of each group were randomly captured and analyzed to evaluate the remodeling of pulmonary artery. CSA and MT + IT were measured by Image-Pro Plus software.

2.15. Immunohistochemistry. Two weeks after ASCs transplantation, heart and lung tissues were collected and fixed in $4 \%$ paraformaldehyde. The primary antibodies were applied to incubate the sections overnight at $4^{\circ} \mathrm{C}$. The secondary antibody was used to cover the tissues for 50 minutes at RT. Immunopositive cells were counted using Image-Pro Plus software. The dilution of antibody was anti-BMPR2 (1:200).

2.16. Statistical Analysis. All experiments were conducted independently at least three times. All data were expressed as the mean \pm SD. Statistical analysis was performed using the GraphPad Prism 6 software. We used Student's t-test to analyze the statistical significance of differences between two groups, and comparisons among three or more groups were made with one-way ANOVA analysis. $\mathrm{P}$ value $<0.05$ was considered statistically significant.

\section{Results}

3.1. Characterization of ASCs Isolated from Adipose Tissue. Fluorescence-activated cell sorting (FACS) analysis showed that cell surface markers CD90 and CD105 were highly expressed while CD31 and CD45 staining was not observed in ASCs (Figure 1(a)). Morphology, adipogenic differentiation, and osteogenic differentiation are shown in Figure 1(b). As expected, at passage 3 most ASCs were adherent and spindle-like after culturing for 7 days. In addition, ASCs formed alizarin-red positive mineral deposits and adipogenic 

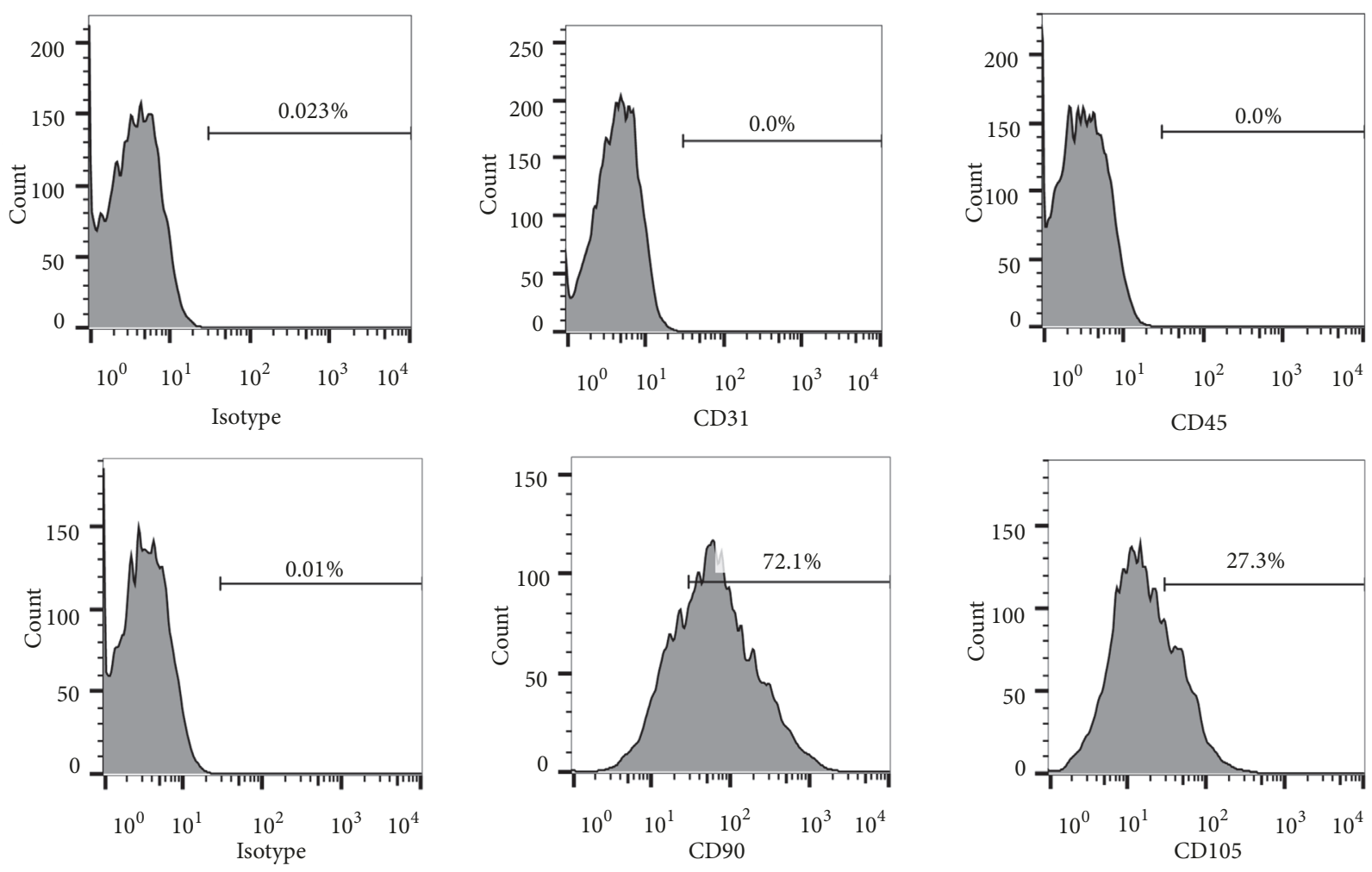

(a)
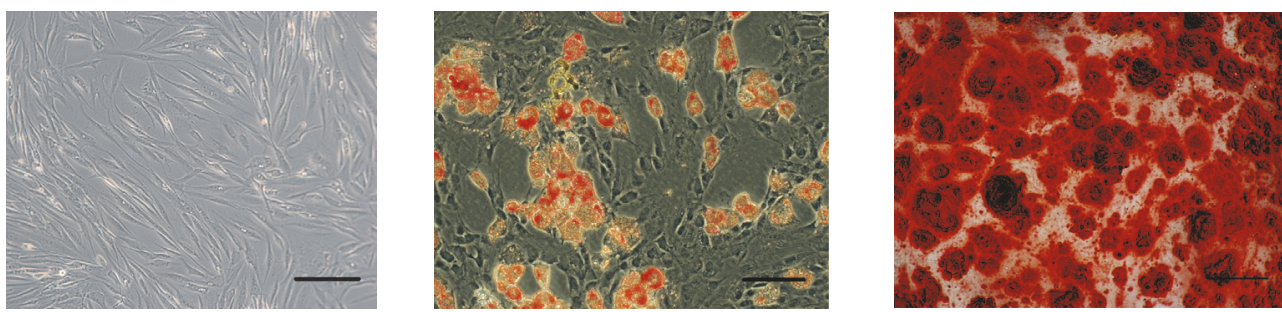

Morphology

Adipogenic differentiation

Osteogenic differentiation

(b)

Conditioned medium

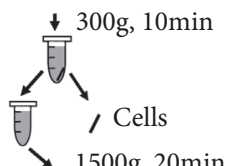

1500g, 20min<smiles>C[I-]1C=C[CH-]1</smiles>

导 / Cell debris

Filtration, $0.22 \mu \mathrm{m}$

E

$\downarrow 150000 \mathrm{~g}, 120 \mathrm{~min}$<smiles>B1CCCCP1</smiles>

E

Wash in PBS $\downarrow 150000 \mathrm{~g}, 120 \mathrm{~min}$

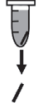

Exosomes

(c)

Figure 1: Continued. 


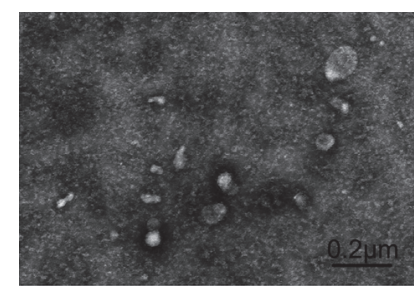

(d)

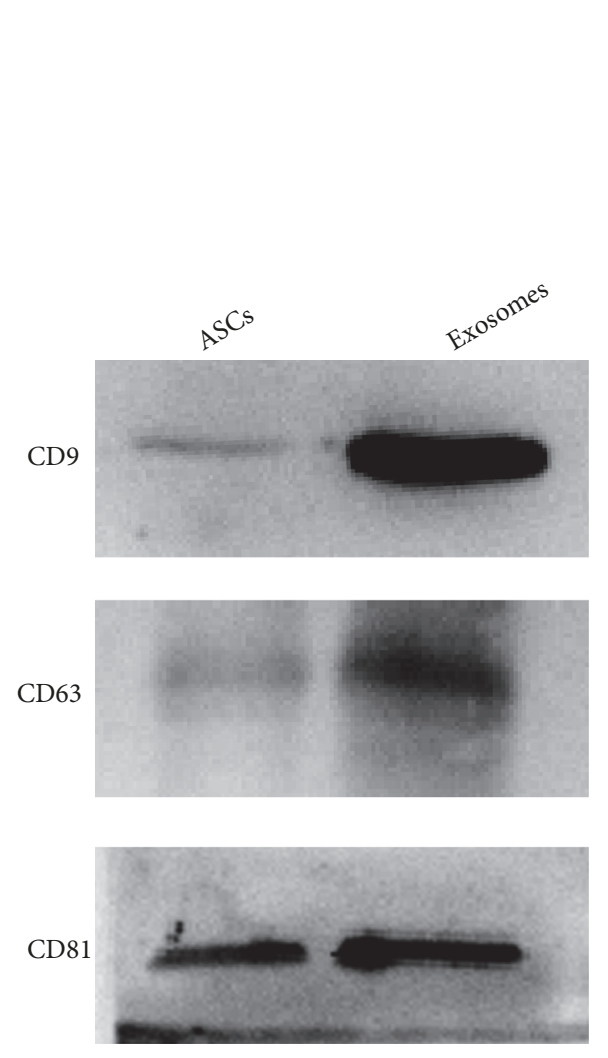

(e)

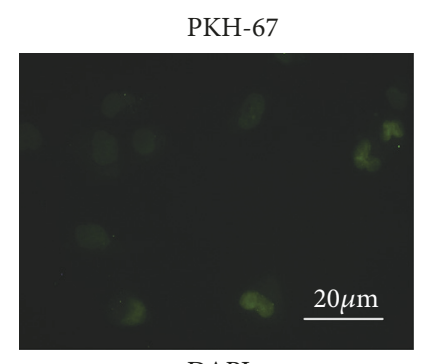

DAPI

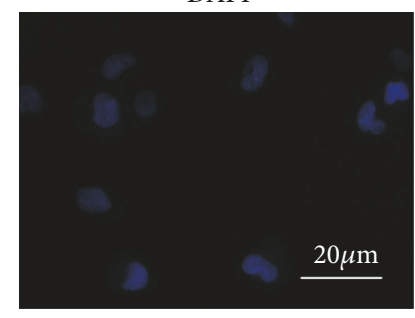

Merge

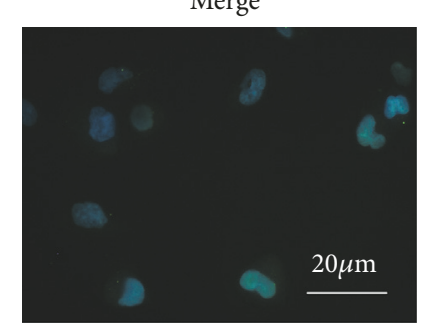

(f)

Figure 1: Characterization of ASCs and ASCs-Exos. (a) The expression level of CD31, CD45, CD90, and CD105 in ASCs was detected by FACS. (b) Morphology, adipogenic differentiation, and osteogenic differentiation of ASCs cultured in vitro. Scale bars $=100 \mu \mathrm{m}$. (c) Scheme of exosomes isolation by ultracentrifugation. (d) Representative electron microscopy image of ASCs-Exos. Scale bars $=0.2 \mu \mathrm{m}$. (e) Western blot analysis of exosomal markers. ASCs protein was used as control for exosomes characterized. (f) ASCs-Exos were labeled with PKH-67. ASCs-Exos transferring into HPAECs were tracked using a fluorescence microscope. Scale bars $=20 \mu \mathrm{m}$.

potential. Therefore, all data suggested successful isolation and characterization of ASCs from adipose tissue.

3.2. ASCs Improved HPAECs Proliferation. Viability of HPAECs incubated with different concentrations of MCTP was assessed by CCK8 assay. Based on significant differences, we selected $60 \mu \mathrm{M}$ MCTP $(\mathrm{P}<0.05)$ for $48 \mathrm{~h}(\mathrm{P}<0.05)$ to treat HPAECs (Figures 2(a) and 2(b)). HPAECs were cocultured with or without ASCs to determine the effect of ASCs on HPAECs. Proliferation of HPAECs decreased significantly after incubating with $60 \mu \mathrm{g} / \mathrm{ml}$ MCTP. However, when cocultured with ASCs, growth of HPAECs was significantly increased (Figures 2(c) and 2(d)). Therefore, we hypothesized that ASCs produce bioactive factors that influence the proliferation of HPAECs.
3.3. ASCs-Exos Improved Cell Proliferation in MCTP-Induced HPAECs. Exosomes were isolated using ultracentrifugation (Figure 1(c)) and identified using transmission electron microscope. We confirmed heterogeneous lipid bilayered vesicles, approximately 50-100 $\mathrm{nm}$ in diameter (Figure $1(\mathrm{~d})$ ). Western blot analysis showed increased expression of exosomal surface markers, CD63, CD9, and CD 81, compared with whole ASCs lysates (Figure 1(e)). HPAECs cocultured with PKH-67-labeled exosomes for 1 hour exhibited high uptake efficiency as detected by fluorescence microscopy (Figure 1(f)). Results, thus, indicated successful isolation of exosomes and their uptake by HPAECs.

To further evaluate the effect of exosomes on HPAECs, HPAECs were cultured with or without exosomes. As shown in Figures 2(e) and 2(f), exosomes improved proliferation of 


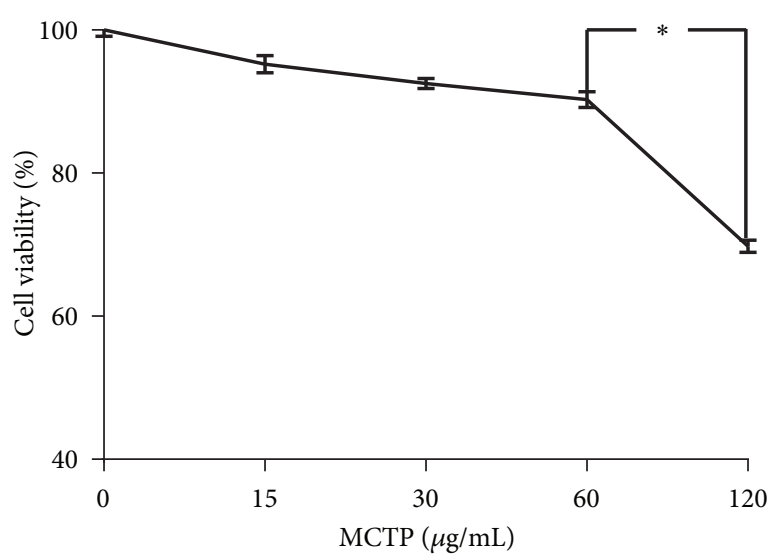

(a)

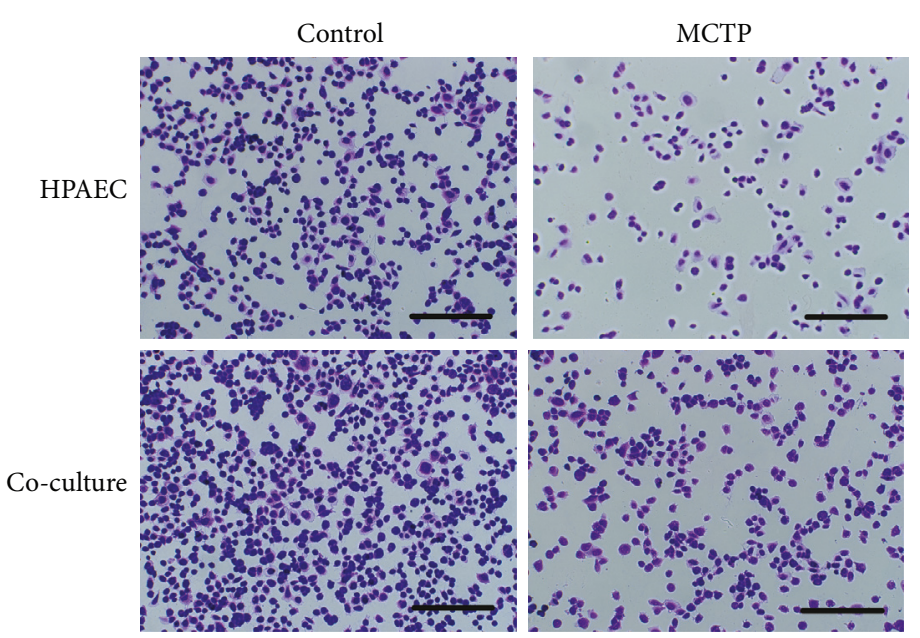

(c)

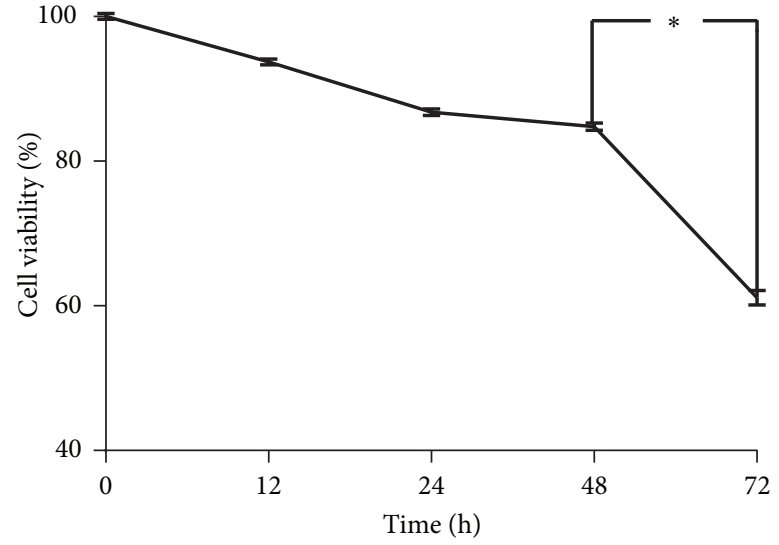

(b)

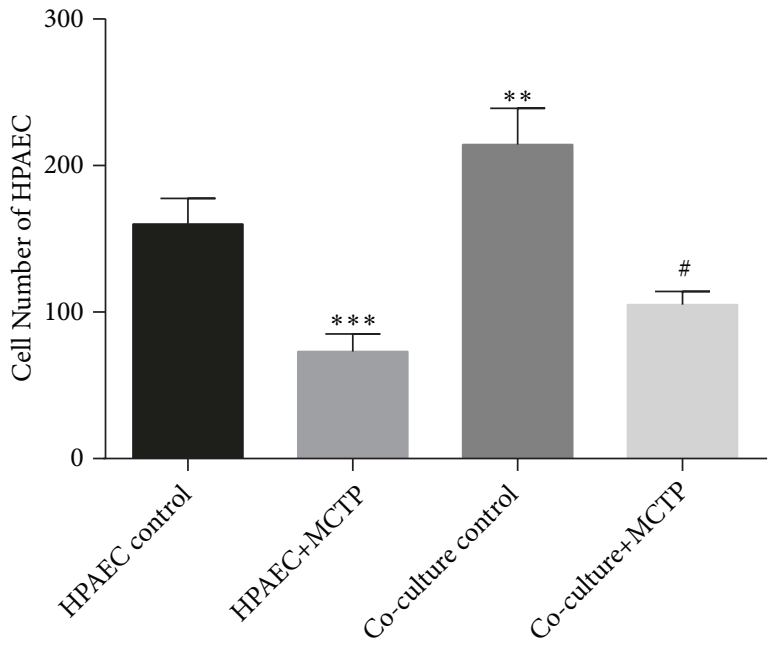

(d)

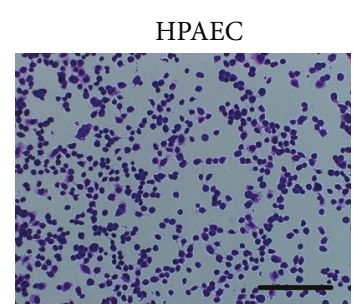

HPAEC+MCTP

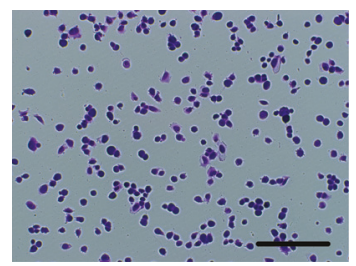

(e)

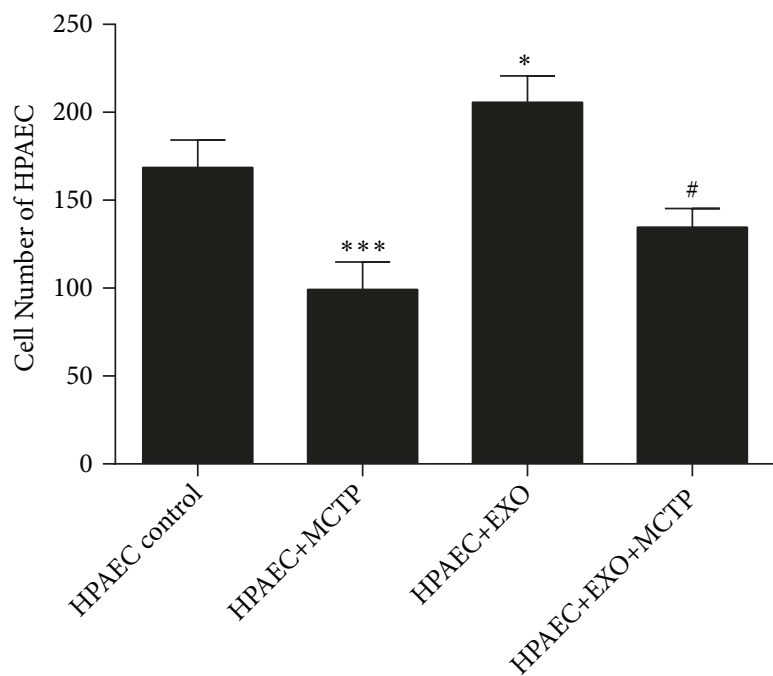

(f)

FIgURE 2: ASCs and ASCs-Exos improved HPAECs proliferation. (a) Cell viability was analyzed by using the CCK8 assay after exposure to MCTP at concentrations of $0,15,30,60$, and $120 \mu \mathrm{M}$ for $24 \mathrm{~h}$. (b) Cell incubation with MCTP for $0,12,24,48$, and $72 \mathrm{~h}$ at the concentration of $60 \mu \mathrm{M}$. (c) Responding pictures of HPAECs cocultured with or without ASCs. Scale bars $=100 \mu \mathrm{m}$. (d) The number of HPAECs, counted in 3 fields under $\times 10$ objective lens. Data are presented as mean $\pm \mathrm{SD}, \mathrm{n}=3 . * * \mathrm{P}<0.01, * * * \mathrm{P}<0.001$ vs. HPAECs control group, ${ }^{\#} \mathrm{P}<0.05$ vs. coculture control group. (e) Representative pictures of HPAECs cultured with or without exosomes. Scale bars $=100 \mu \mathrm{m}$. (f) The number of HPAECs cultured with or without exosomes, counted in 3 fields under $\times 10$ objective lens. Data are presented as mean $\pm \mathrm{SD}, \mathrm{n}=3 . * \mathrm{P}<$ $0.05, * * * \mathrm{P}<0.001$ vs. HPAECs group, ${ }^{*} \mathrm{P}<0.05$ vs. HPAECs+MCTP group. 


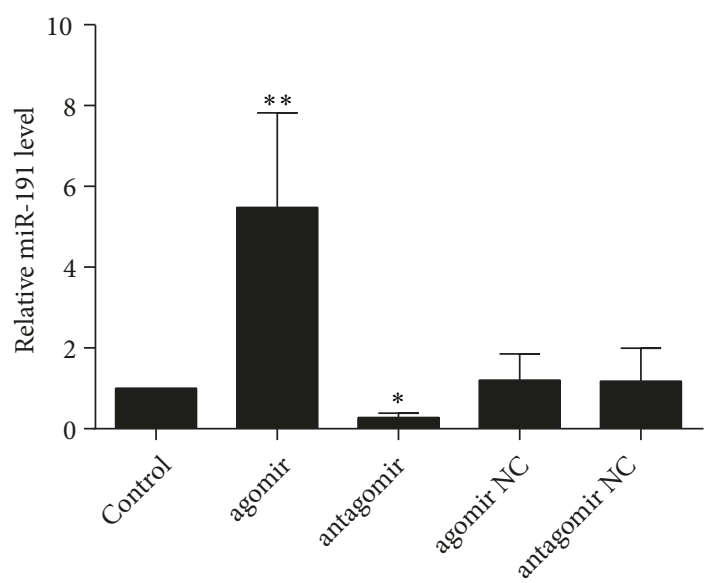

(a)

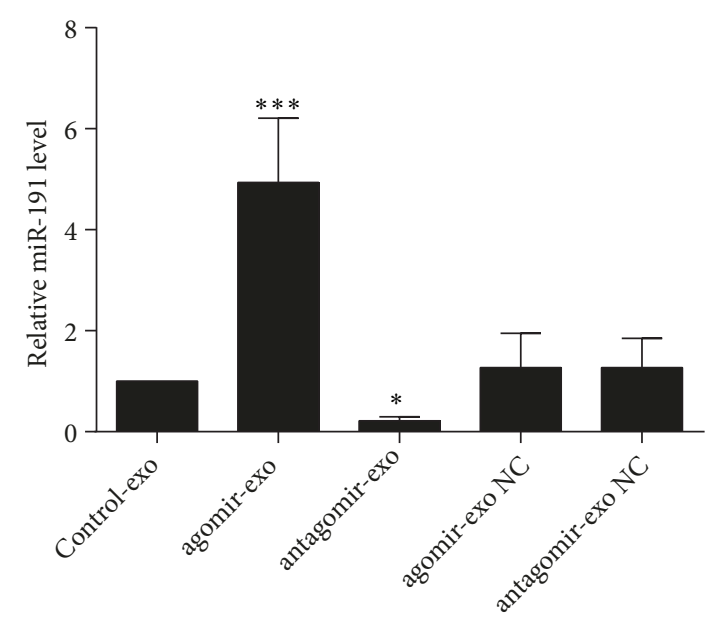

(b)
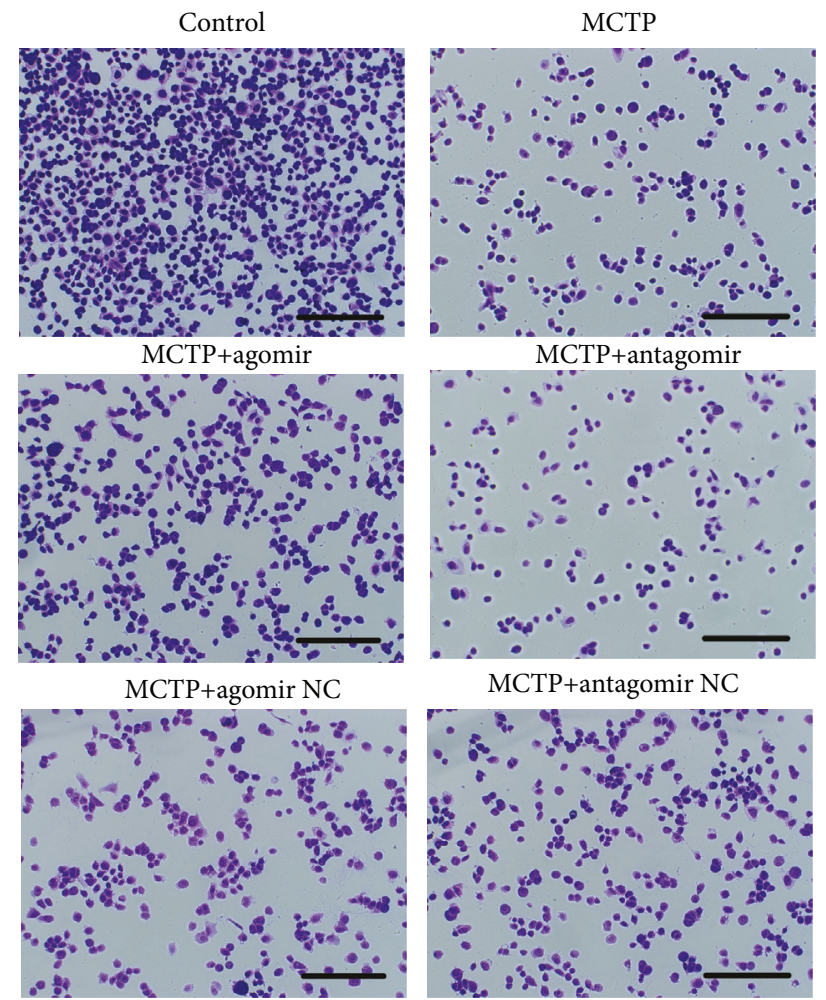

MCTP+antagomir NC

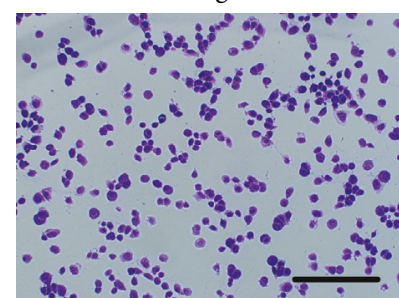

(c)

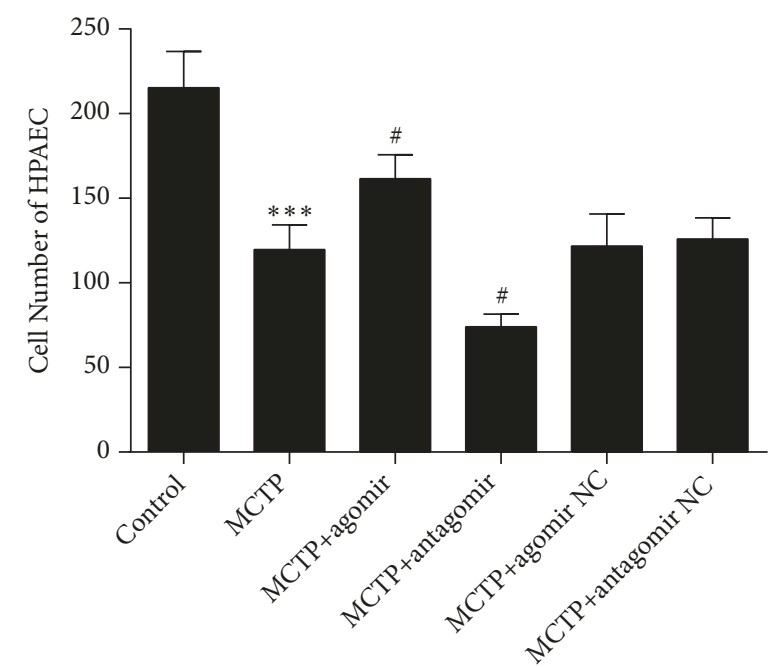

(d)

Figure 3: ASCs-Exos containing miR-191 affected HPAECs proliferation. (a, b) The expression levels of miR-191 in ASCs and exosomes. Data was presented as the mean $\pm \mathrm{SD}, \mathrm{n}=3 . * \mathrm{P}<0.05, * * \mathrm{P}<0.01$ vs. control group. (c) Representative pictures of HPAECs cultured with exosomes that ASCs transfected with agomir or antagomir. Scale bars $=100 \mu \mathrm{m}$. (d) The number of HPAECs in (c), counted in 3 fields under $\times 10$ objective lens. Data are presented as mean $\pm \mathrm{SD}, \mathrm{n}=3 . * * * \mathrm{P}<0.001$ vs. control group, ${ }^{\#} \mathrm{P}<0.05$ vs. MCTP group.

both HPAECs and MCTP-treated HPAECs. Results showing that ASCs-Exos modulated HPAECs proliferation, in turn, supported our conjecture that ASCs secreted certain bioactive factors to affect HPAECs proliferation.

3.4. Circulating Exosomes Containing miR-191 Affected Proliferation by Targeting BMPR2. Real-time PCR (RT-PCR) was performed to quantify the relative levels of miR-191 in ASCs and exosomes after transfection of miR-191, showing that miR-191 was enriched in ASCs and ASCs-Exos after transfection with agomir; meanwhile, miR-191 was poorly expressed after transfection with antagomir (Figures 3(a) and 3(b)). Next, we found that high exosomal miR-191 markedly accelerated HPAECs proliferation, whereas low exosomal miR-191 attenuated them (Figures 3(c) and 3(d)). These results collectively suggested that exosomes expressing different levels of miR-191 regulate HPAECs proliferation. 

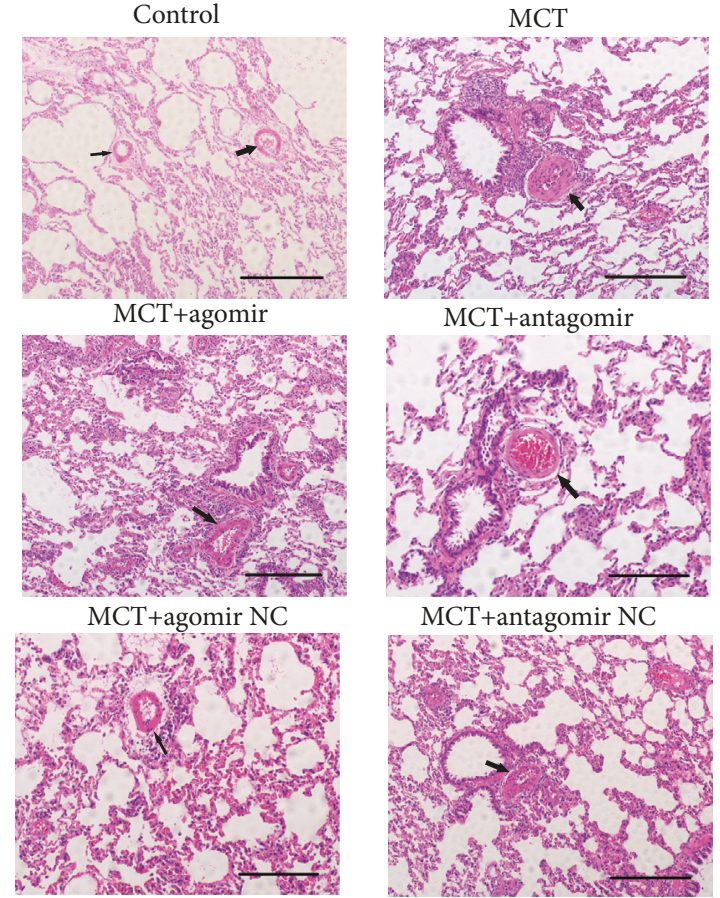

(a)
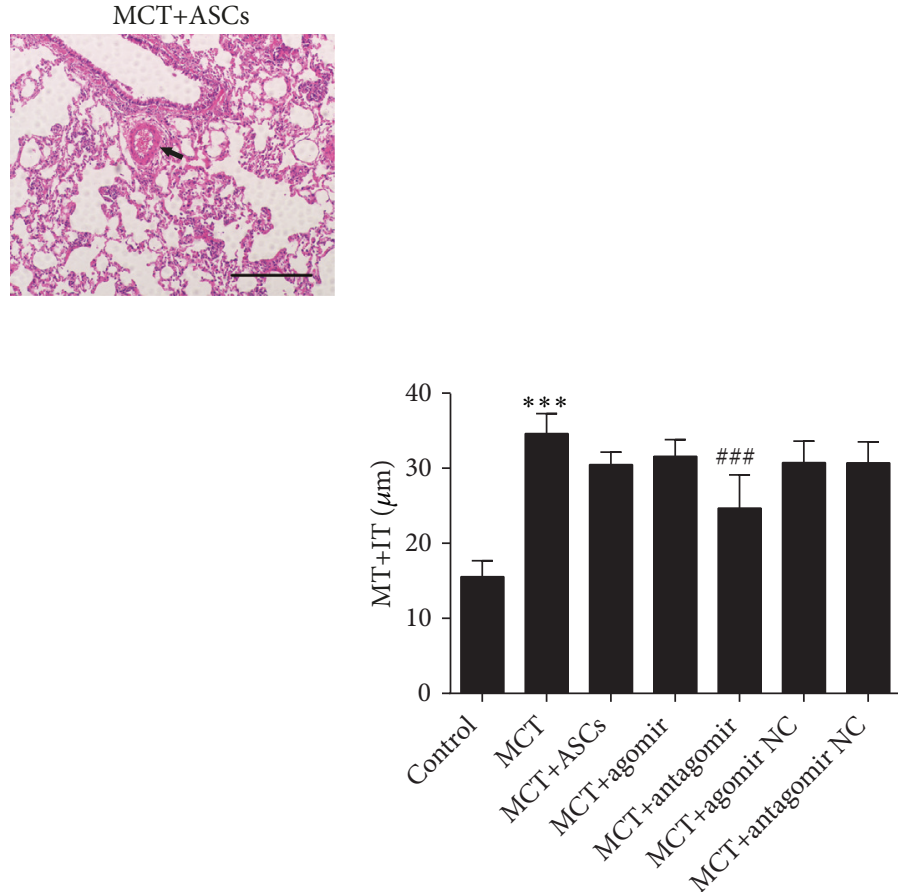

(b)

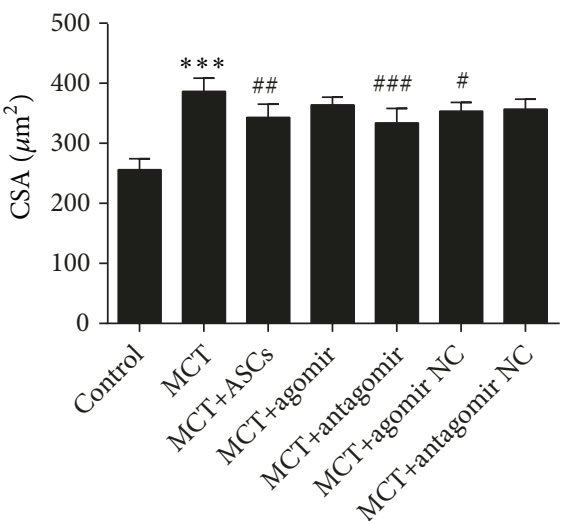

(c)

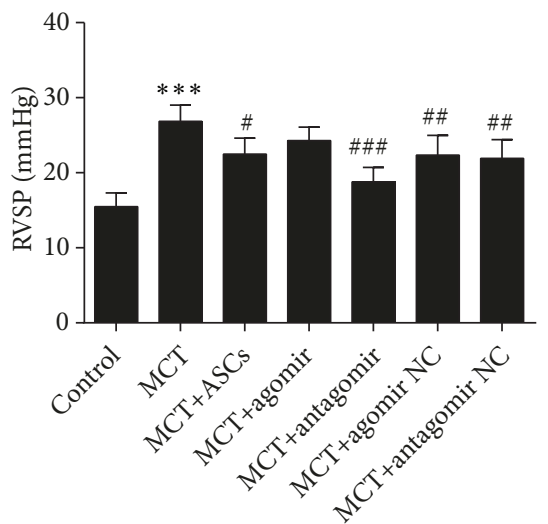

(d)

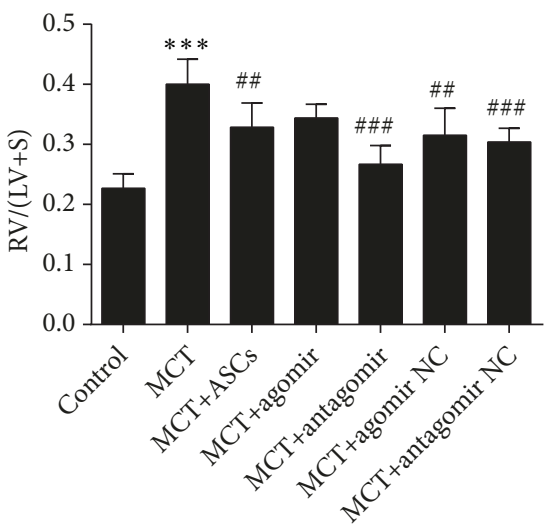

(e)

FIGURE 4: ASCs ameliorated pulmonary and heart function in a rat PAH model. (a) Representative H\&E staining pictures of pulmonary artery vascular. Scale bars $=100 \mu \mathrm{m}$. (b-e) The comparison of ET+MT, CSA, RVSP, and RV/(LV+S) after different ASCs administration for 2 weeks. Data was presented as the mean $\pm \mathrm{SD}, \mathrm{n}=5-6 . * * * \mathrm{P}<0.001$ vs. control group, ${ }^{\#} \mathrm{P}<0.05,{ }^{\# \#} \mathrm{P}<0.01$, ${ }^{\# \#} \mathrm{P}<0.001$ vs. MCT group.

Posttranscriptional gene regulation is the major biological function of miRNA. In order to identify downstream targets of miR-191, we investigated BMPR2 expression in HPAECs. Expression of BMPR2 was analyzed by western blotting (Figure 5(a)). The result suggested that expression of BMPR2 protein in HPAECs was remarkably increased in the antagomir group and decreased in the MCT and agomir groups.

3.5. ASCs Ameliorated Pulmonary and Heart Function in a Rat PAH Model through miR-191. ASCs with high or low miR-191 expression were obtained after transfection with miR-191 agomir or antagomir, respectively (Figure 3(a)). Two weeks following MCT administration, ASCs with enriched or diminished miR-191 expression and corresponding negative control (NC) were injected via intraperitoneal vein to $\mathrm{PAH}$ rats. Medial wall thickness plus intimal thickness (MT + IT), Cross-sectional area (CSA), RVSP, and right ventricle (RV)-to-left ventricle (LV) + septum $(\mathrm{S})(\mathrm{RV} / \mathrm{LV}+\mathrm{S})$ values after two weeks of ASCs administration are shown in Figures 4(b)-4(e). RVSP was significantly increased in the MCT group compared with the control group $(26.85 \pm 1.94 \mathrm{mmHg}$ vs. $15.47 \pm 1.69 \mathrm{mmHg} ; \mathrm{p}<0.001)$. In contrast, compared with the MCT group, two weeks after ASCs administration, RVSP was notably reduced which was further lowered in the antagomir group $(18.77 \pm 1.76 \mathrm{mmHg}$ vs. $26.85 \pm 1.94 \mathrm{mmHg}$; $\mathrm{p}<0.001)$. Similar result was observed for $\mathrm{RV} /(\mathrm{LV}+\mathrm{S})$. The $\mathrm{RV} /(\mathrm{LV}+\mathrm{S})$ was much higher in the MCT group than 


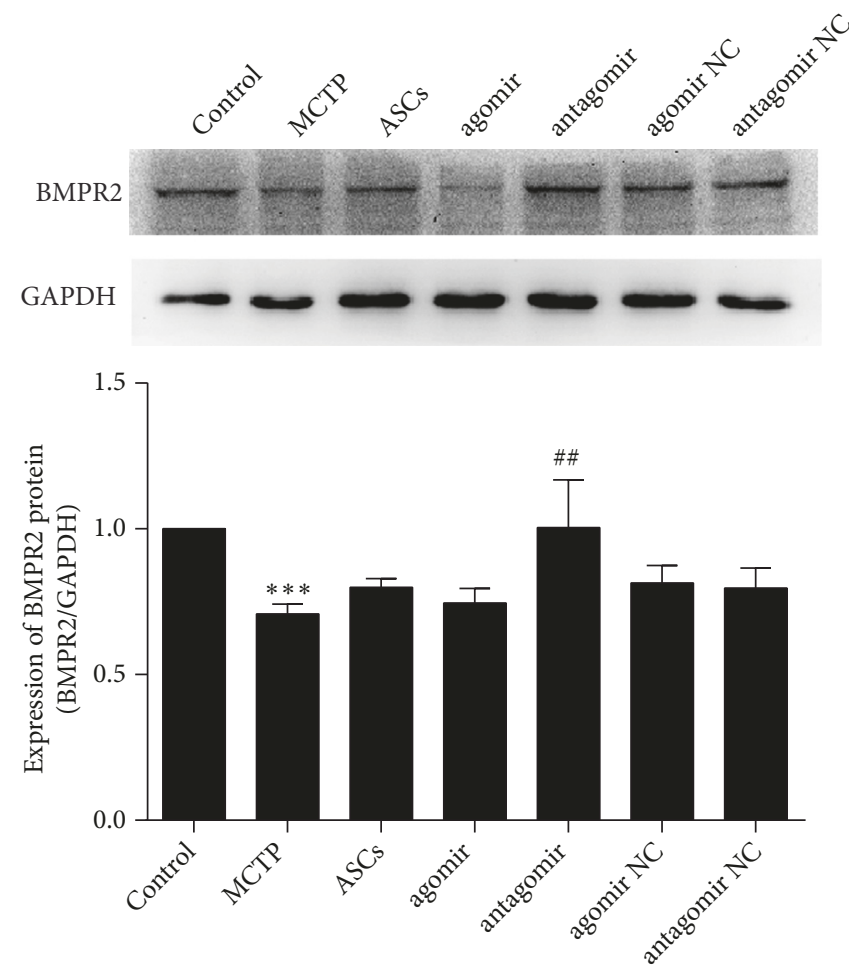

(a)
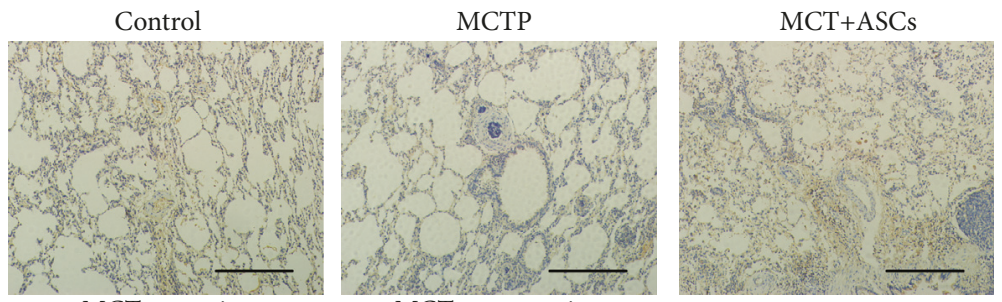

MCT+agomir

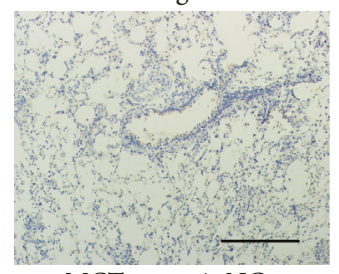

MCT+antagomir

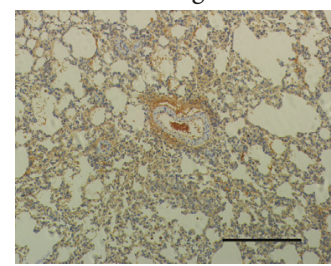

MCT+agomir NC
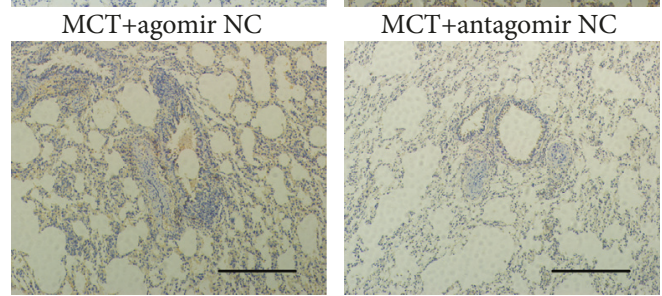

(b)

FIGURE 5: miR-191 directly targets BMPR2 in HPAECs and PAH rats. (a) The protein expression of BMPR2 compared with GAPDH (data is presented as the mean \pm SD) in HPAECs. $* * * \mathrm{P}<0.001$ vs. control group, ${ }^{\# \#} \mathrm{P}<0.01$ vs. MCTP group. (b) Immunohistochemistry staining results for BMPR2 in lungs of rats. Scale bars $=100 \mu \mathrm{m}$.

in the control group $(0.4 \pm 0.04$ vs. $0.23 \pm 0.02 ; \mathrm{p}<$ $0.001)$. Nonetheless, two weeks after treatment, RV/(LV+S) significantly decreased in the antagomir group, much lower than the MCT group $(0.27 \pm 0.03$ vs. $0.4 \pm 0.04 ; \mathrm{p}<$
0.001). To further evaluate the effect of ASCs, right lungs from $\mathrm{PAH}$ rats were stained by routine hematoxylin and eosin (H\&E) staining (Figure 4(a)). As shown in Figure 4(b), $\mathrm{MT}+\mathrm{IT}$ was much higher in the MCT group than in the 
control group $(15.54 \pm 1.97 \mu \mathrm{m}$ vs. $34.63 \pm 2.41 \mu \mathrm{m} ; \mathrm{p}<0.001)$. Similarly, administration of ASCs significantly decreased MT+IT compared with the MCT group. MT+IT in the miR191 antagomir group was much lower than the MCT group $(24.67 \pm 4.04 \mu \mathrm{m}$ vs. $34.63 \pm 2.41 \mu \mathrm{m} ; \mathrm{p}<0.001)$. CSA was significantly increased in the MCT group compared with the control group $\left(386.33 \pm 20.12 \mu \mathrm{m}^{2}\right.$ vs. $256.17 \pm 16.80 \mu \mathrm{m}^{2} ; \mathrm{p}<$ 0.001 , Figure $4(\mathrm{c}))$. ASCs administration, however, reduced CSA. Moreover, CSA in the antagomir group was much lower than in the MCT group $\left(334 \pm 21.92 \mu \mathrm{m}^{2}\right.$ vs. $386.33 \pm$ $\left.20.12 \mu \mathrm{m}^{2} ; \mathrm{p}<0.001\right)$. This evidence suggested miR-191 as a potential risk factor for the development of PAH in rats.

In order to identify downstream targets of miR-191 in PAH rats, we investigated BMPR2 expression in rat lungs. BMPR2 expression was high in the antagomir group and poorly expressed in the MCT group as assessed by immunohistochemistry (Figure 5(b)). On the basis of this result, we found that miR-191 directly affected BMPR2 expression in PAH rats.

\section{Discussion}

Excessive proliferation of HPAECs is one of the major pathological features of vasculature remodeling, and inhibition of excessive proliferation of endothelial cells is a vital element of current PAH therapies $[25,26]$. In vitro, we treated HPAECs with MCTP, an alkylating agent that is synthesized from MCT in the liver [27]. Our finding that MCTP inhibited HPAECs proliferation is in line with previous studies reporting that MCTP-induced protein and DNA adducts ultimately were responsible for endothelial cell cycle arrest [28-31]. Next, we explored the effect of ASCs on MCTP-treated HPAECs and observed that ASCs improved proliferation of MCTP-treated HPAECs.

Recently, aberrant expression of miR-191 has been reported to be associated with various cancers $(>20)$ and other diseases including Crohn's, type- 2 diabetes, pulmonary hypertension, and Alzheimer's [32]. A recent study has corroborated that miR-191 was one of upregulated miRNAs in the circulation of $\mathrm{PH}$ subjects [22]. Interestingly, miR191 is one of the most represented miRNAs in ASCs-Exos [21]. Compelling reports indicate that exosomes released from ASCs have a therapeutic role through transfer of characteristic proteins, mRNA, as well as miRNAs [33-35]. To elucidate the underlying mechanism of ASCs mediated protection of HPAECs, ASCs-Exos were incubated with MCTP-treated HPAECs. Interestingly, in the presence of the miR-191 antagomir, we observed a significant reversal of protective effect and vice versa with the miR-191 agomir. Thus, these results demonstrated that ASCs-Exos, at least in part, influenced proliferation of HPAECs via miR-191, which subsequently might affect the progression of vasculature remodeling.

$\mathrm{PAH}$ is characterized by functional and structural changes in the pulmonary vasculature, and thus it is critical to terminate or reverse the ensuing remodeling of vasculature $[3,9]$. ASCs transplantation is considered to be a potential therapeutic choice. Previous studies have shown that
ASCs possess the potential to reverse pulmonary arteriole remodeling and hypertrophy of the right ventricle [3638]. However, the specific mechanisms underlying the ASCs mediated reversal of remodeling are unclear. The MCTinduced rat PAH model, commonly used by researchers, is an inexpensive and reproducible tool that does not require meticulous technical skills [39]. In this study, we utilized this model to explore the influence of miR-191-modified ASCs on PAH. Data showed that miR-191 antagomir induced a significant reversal of vascular remodeling, and downregulation of mediators associated with pulmonary hypertension, suggesting that this effect was, in part, modulated by miR191. Understanding the mechanisms responsible for miR-191related vasculature remodeling, thus, may reveal additional strategies for therapeutic intervention.

That BMPR2 signaling plays a critical role in the pathogenesis of $\mathrm{PAH}$, which is evident from previous studies that showed deletion of BMPR2 in endothelial cells induced PAH in mice [40]. Furthermore, overexpression of miR-191 is associated with pulmonary hypertension [22]. Therefore, we explored the involvement of BMPR2 in miR-191 mediated enhancement of growth of HPAECs. Consistently with our previous hypothesis, miR-191 significantly decreased BMPR2 level. In the presence of the miR-191 antagomir, BMPR2 level increased, with corresponding improvement in symptoms of PAH. Therefore, BMPR2 was involved in miR-191-regulated survival of HPAECs.

\section{Conclusion}

Our study showed that inhibition of miR-191 could ameliorate the development of MCT-induced PAH possibly via preventing BMPR2 degradation. Moreover, BMPR2 was involved in miR-191 mediated HPAECs proliferation. Taken together, we found that miR-191 could be a potential risk factor for PAH. Therefore, this study provided the basic insight into the use of anti-miR-191 as a therapeutic strategy against PAH. However, this is a preliminary study to unravel the possible mechanism of miR-191 and its application in PAH which warrants further studies to validate the above findings in patients. This study only illuminates the effect of miR-191 on HPAECs and rat PAH model. The next step would be to explore the exact mechanisms for application in human therapy.

\section{Data Availability}

The data used to support the findings of this study are available from the corresponding author upon request.

\section{Conflicts of Interest}

All authors have no conflicts of interest.

\section{Authors' Contributions}

Caixin Zhang and Pengbo Wang equally contributed to this work. 


\section{Acknowledgments}

This work was supported by grants from Nantong Science and Technology Plan-Key technology research (No. MS32015025); Youth Subject of Jiangsu Provincial Commission of Health and Family Planning (No. Q2017009); and Six Talent Peaks project in Jiangsu Province (No. WSN-062; No. WSW-049).

\section{References}

[1] N. Galiè, M. Humbert, J.-L. Vachiery et al., “2015 ESC/ERS guidelines for the diagnosis and treatment of pulmonary hypertension: The joint task force for the diagnosis and treatment of pulmonary hypertension of the european society of cardiology (ESC) and the european respiratory society (ERS): Endorsed by: Association for european paediatric and congenital cardiology (AEPC), international society for heart and lung transplantation (ISHLT)," European Heart Journal, vol. 37, no. 1, pp. 67-119, 2016.

[2] M. Guazzi and R. Naeije, "Pulmonary hypertension in heart failure: pathophysiology, pathobiology, and emerging clinical perspectives," Journal of the American College of Cardiology, vol. 69, no. 13, pp. 1718-1734, 2017.

[3] N. W. Morrell, S. Adnot, S. L. Archer et al., "Cellular and molecular basis of pulmonary arterial hypertension," Journal of the American College of Cardiology, vol. 54, no. 1, pp. S20-S31, 2009.

[4] S. Malenfant, A.-S. Neyron, R. Paulin et al., "Signal transduction in the development of pulmonary arterial hypertension," Pulmonary Circulation, vol. 3, no. 2, pp. 278-293, 2013.

[5] A. T. J. Maria, K. Toupet, M. Maumus et al., "Human adipose mesenchymal stem cells as potent anti-fibrosis therapy for systemic sclerosis," Journal of Autoimmunity, vol. 70, pp. 31-39, 2016.

[6] M. F. Pittenger, A. M. Mackay, S. C. Beck et al., "Multilineage potential of adult human mesenchymal stem cells," Science, vol. 284, no. 5411, pp. 143-147, 1999.

[7] Y. Miyahara, N. Nagaya, M. Kataoka et al., "Monolayered mesenchymal stem cells repair scarred myocardium after myocardial infarction," Nature Medicine, vol. 12, no. 4, pp. 459-465, 2006.

[8] N. H. Nicolay, R. Lopez Perez, J. Debus, and P. E. Huber, "Mesenchymal stem cells-a new hope for radiotherapy-induced tissue damage?" Cancer Letters, vol. 366, no. 2, pp. 133-140, 2015.

[9] Y. Fu, L. Karbaat, L. Wu, J. Leijten, S. K. Both, and M. Karperien, "Trophic effects of mesenchymal stem cells in tissue regeneration," Tissue Engineering - Part B: Reviews, vol. 23, no. 6, pp. 515-528, 2017.

[10] C. Lee, S. A. Mitsialis, M. Aslam et al., "Exosomes mediate the cytoprotective action of mesenchymal stromal cells on hypoxiainduced pulmonary hypertension," Circulation, vol. 126, no. 22, pp. 2601-2611, 2012.

[11] L. Luo, W. Zheng, G. Lian et al., "Combination treatment of adipose-derived stem cells and adiponectin attenuates pulmonary arterial hypertension in rats by inhibiting pulmonary arterial smooth muscle cell proliferation and regulating the AMPK/BMP/Smad pathway," International Journal of Molecular Medicine, vol. 41, no. 1, pp. 51-60, 2018.

[12] B.-T. Pan, K. Teng, C. Wu, M. Adam, and R. M. Johnstone, "Electron microscopic evidence for externalization of the transferrin receptor in vesicular form in sheep reticulocytes," The Journal of Cell Biology, vol. 101, no. 3, pp. 942-948, 1985.

[13] J. Skog, T. Würdinger, S. van Rijn et al., "Glioblastoma microvesicles transport RNA and proteins that promote tumour growth and provide diagnostic biomarkers," Nature Cell Biology, vol. 10, no. 12, pp. 1470-1476, 2008.

[14] B. Dörsam, K. S. Reiners, and E. P. von Strandmann, "Cancerderived extracellular vesicles: Friend and foe of tumour immunosurveillance," Philosophical Transactions of the Royal Society B: Biological Sciences, vol. 373, no. 1737, Article ID 20160481, 2018.

[15] J. Chen, C. Hu, and P. Pan, "Extracellular vesicle microRNA transfer in lung diseases," Frontiers in Physiology, vol. 8, p. 1028, 2017.

[16] F. Pardo, R. Villalobos-Labra, B. Sobrevia, F. Toledo, and L. Sobrevia, "Extracellular vesicles in obesity and diabetes mellitus," Molecular Aspects of Medicine, vol. 60, pp. 81-91, 2018.

[17] D. Schatz and A. Vardi, "Extracellular vesicles - new players in cell-cell communication in aquatic environments," Current Opinion in Microbiology, vol. 43, pp. 148-154, 2018.

[18] A. E. Sedgwick and C. D’Souza-Schorey, “The biology of extracellular microvesicles," Traffic, vol. 19, no. 5, pp. 319-327, 2018.

[19] M. R. Fabian, N. Sonenberg, and W. Filipowicz, "Regulation of mRNA translation and stability by microRNAs," Annual Review of Biochemistry, vol. 79, pp. 351-379, 2010.

[20] G. Di Leva, M. Garofalo, and C. M. Croce, "MicroRNAs in cancer," Annual Review of Pathology: Mechanisms of Disease, vol. 9, pp. 287-314, 2014.

[21] S. R. Baglio, K. Rooijers, D. Koppers-Lalic et al., "Human bone marrow- and adipose-mesenchymal stem cells secrete exosomes enriched in distinctive miRNA and tRNA species," Stem Cell Research \& Therapy, vol. 6, no. 1, article 127, 2015.

[22] C. Wei, H. Henderson, C. Spradley et al., "Circulating miRNAs as potential marker for pulmonary hypertension," PLoS ONE, vol. 8, no. 5, Article ID e64396, 2013.

[23] B. T. Estes, B. O. Diekman, J. M. Gimble, and F. Guilak, "Isolation of adipose-derived stem cells and their induction to a chondrogenic phenotype," Nature Protocols, vol. 5, no. 7, pp. 1294-1311, 2010.

[24] A. R. Mattocks, J. Rebekah, and B. Janet, "Simple procedures for preparing putative toxic metabolites of pyrrolizidine alkaloids," Toxicon, vol. 27, no. 5, pp. 561-567, 1989.

[25] M. Rabinovitch, "Molecular pathogenesis of pulmonary arterial hypertension," The Journal of Clinical Investigation, vol. 122, no. 12, pp. 4306-4313, 2012.

[26] S. L. Archer, E. K. Weir, and M. R. Wilkins, "Basic science of pulmonary arterial hypertension for clinicians: new concepts and experimental therapies," Circulation, vol. 121, no. 18, pp. 2045-2066, 2010.

[27] M. Shah, K. Patel, and P. B. Sehgal, "Monocrotaline pyrroleinduced endothelial cell megalocytosis involves a Golgi blockade mechanism," American Journal of Physiology-Cell Physiology, vol. 288, no. 4, pp. C850-C862, 2005.

[28] M. Ramos, M. W. Lamé, H. J. Segall, and D. W. Wilson, "Monocrotaline pyrrole induces Smad nuclear accumulation and altered signaling expression in human pulmonary arterial endothelial cells," Vascular Pharmacology, vol. 46, no. 6, pp. 439-448, 2007.

[29] H. C. Thomas, M. W. Lamé, D. W. Wilson, and H. J. Segall, "Cell cycle alterations associated with covalent binding of 
monocrotaline pyrrole to pulmonary artery endothelial cell DNA," Toxicology and Applied Pharmacology, vol. 141, no. 1, pp. 319-329, 1996.

[30] D. W. Wilson, M. W. Lamé, S. K. Dunston, D. W. Taylor, and H. J. Segall, "Monocrotaline pyrrole interacts with actin and increases thrombin- mediated permeability in pulmonary artery endothelial cells," Toxicology and Applied Pharmacology, vol. 152, no. 1, pp. 138-144, 1998.

[31] R. Mathew, J. Huang, M. Shah, K. Patel, M. Gewitz, and P. B. Sehgal, "Disruption of endothelial-cell caveolin- $1 \alpha / \mathrm{raft}$ scaffolding during development of monocrotaline-induced pulmonary hypertension," Circulation, vol. 110, no. 11, pp. 14991506, 2004.

[32] N. Nagpal and R. Kulshreshtha, "miR-191: an emerging player in disease biology," Frontiers in Genetics, vol. 5, article 99, 2014.

[33] L. Wang, L. Hu, X. Zhou et al., "Exosomes secreted by human adipose mesenchymal stem cells promote scarless cutaneous repair by regulating extracellular matrix remodelling," Scientific Reports, vol. 7, no. 1, p. 13321, 2017.

[34] C. Ding, Q. Zou, F. Wang et al., "HGF and BFGF secretion by human adipose-derived stem cells improves ovarian function during natural aging via activation of the SIRT1/FOXO1 signaling pathway," Cellular Physiology and Biochemistry, vol. 45, no. 4, pp. 1316-1332, 2018.

[35] J. M. Aliotta, M. Pereira, S. Wen et al., "Exosomes induce and reverse monocrotaline-induced pulmonary hypertension in mice," Cardiovascular Research, vol. 110, no. 3, pp. 319-330, 2016.

[36] K. Liu, R. Liu, G. Cao, H. Sun, X. Wang, and S. Wu, "Adiposederived stromal cell autologous transplantation ameliorates pulmonary arterial hypertension induced by shunt flow in rat models," Stem Cells and Development, vol. 20, no. 6, pp. 10011010, 2011.

[37] N. K. Somanna, P. M. Wörner, S. N. Murthy et al., "Intratracheal administration of cyclooxygenase-1-transduced adipose tissuederived stem cells ameliorates monocrotaline-induced pulmonary hypertension in rats," American Journal of PhysiologyHeart and Circulatory Physiology, vol. 307, no. 8, pp. H1187H1195, 2014.

[38] A. Rathinasabapathy, E. Bruce, A. Espejo et al., "Therapeutic potential of adipose stem cell-derived conditioned medium against pulmonary hypertension and lung fibrosis," British Journal of Pharmacology, pp. 2859-2879, 2016.

[39] J. G. Gomez-Arroyo, L. Farkas, A. A. Alhussaini et al., “The monocrotaline model of pulmonary hypertension in perspective," American Journal of Physiology-Lung Cellular and Molecular Physiology, vol. 302, no. 4, pp. L363-L369, 2012.

[40] K.-H. Hong, Y. J. Lee, E. Lee et al., "Genetic ablation of the Bmpr2 gene in pulmonary endothelium is sufficient to predispose to pulmonary arterial hypertension," Circulation, vol. 118, no. 7, pp. 722-730, 2008. 


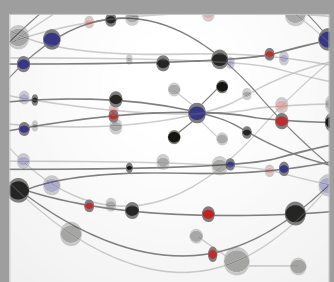

The Scientific World Journal
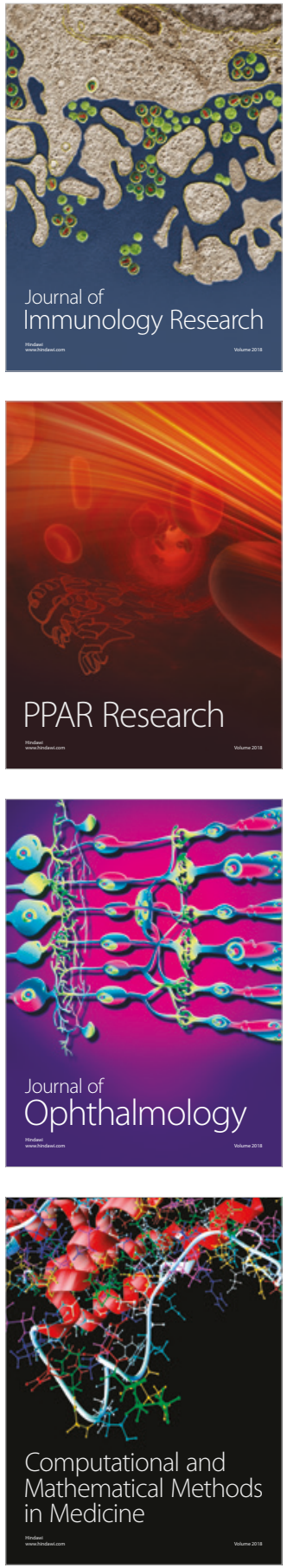

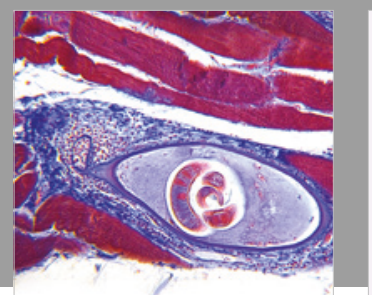

Gastroenterology Research and Practice

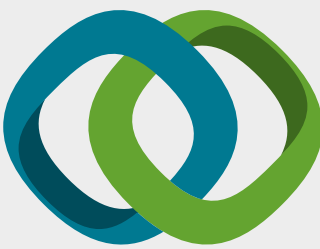

\section{Hindawi}

Submit your manuscripts at

www.hindawi.com
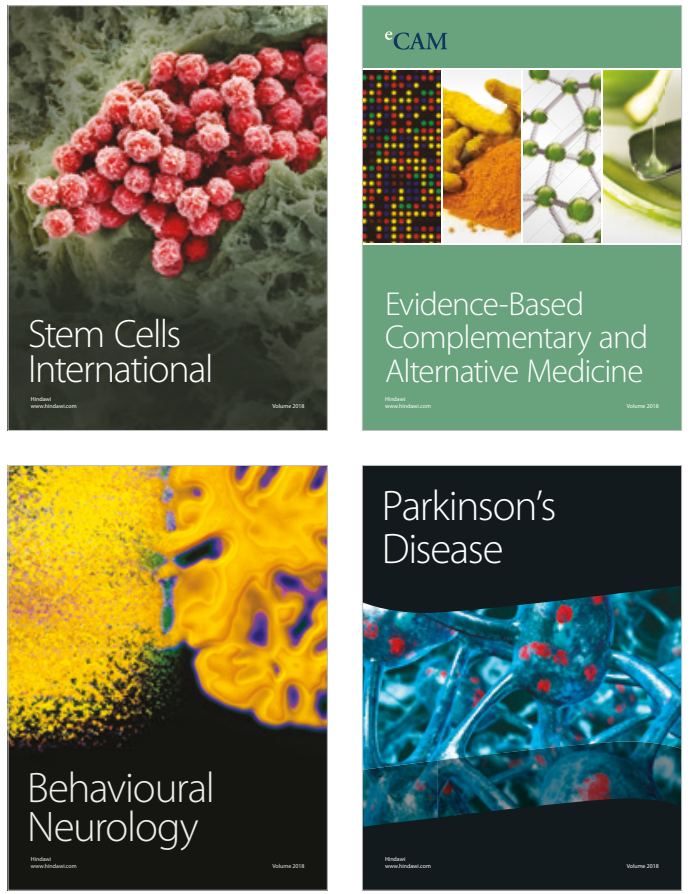

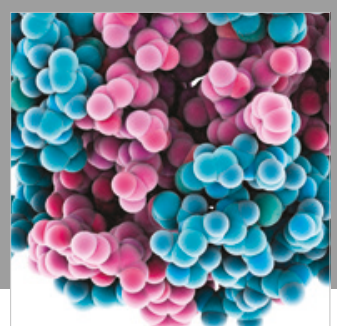

ournal of

Diabetes Research

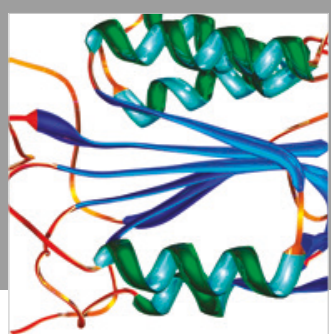

Disease Markers
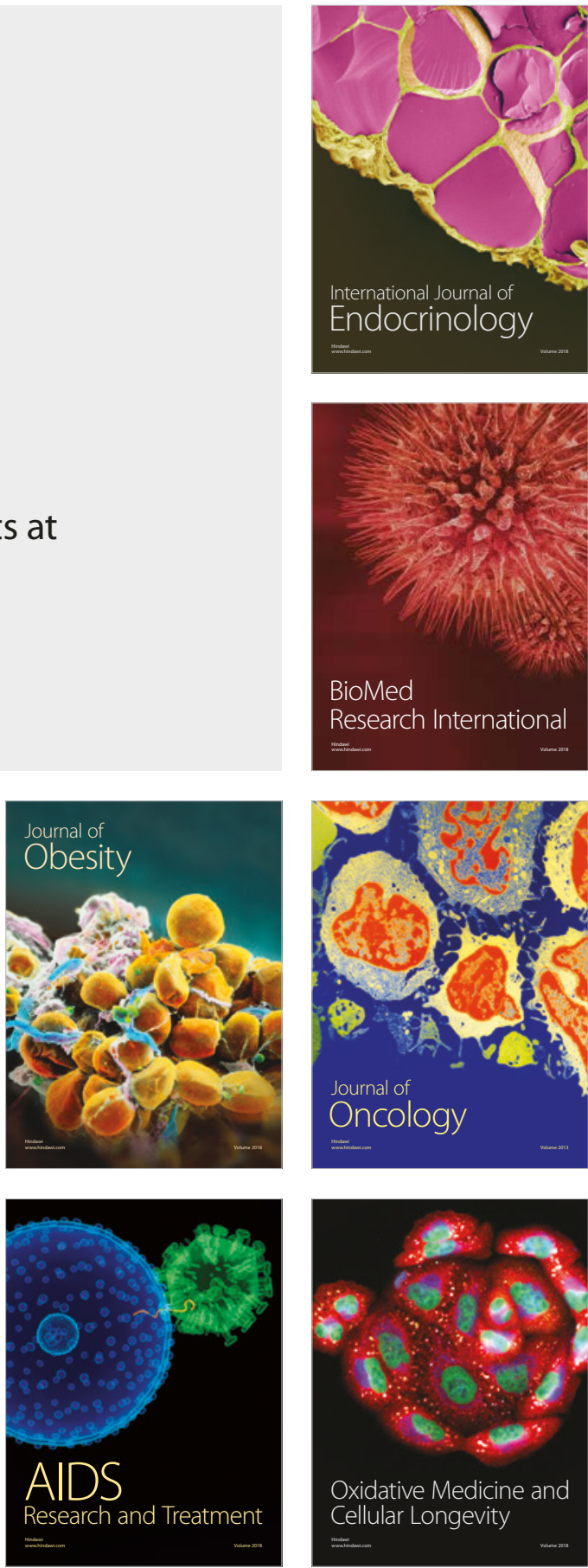